Emmanouil Dermitzakis and Stylianos Antonarakis, now both at the University of Geneva Medical School in Switzerland, and their team broke this down further by looking at how gene expression in different cell types derived from 75 people correlated with variations in their genomes. They took umbilical cord blood from pregnant women and cultured three types of cell for each. Comparing expression between individuals for each cell type, they found that $69-80 \%$ of gene variants affect expression levels in a manner specific to the cell type, suggesting that looking at just one tissue type is insufficient when comparing individuals.

\section{WATER MANAGEMENT}

\section{Colorado be dammed}

Water Resources Res. doi:10.1029/2008WR007652 (2009)

By 2057, climate change could cause a tenfold increase in the annual risk of water shortages in the southwestern United States, say Balaji Rajagopalan at the University of Colorado in Boulder and his colleagues. Rajagopalan's team modelled a variety of management and climate scenarios for varying levels of potential demand over the period 2008-57 for the dam-created Lakes Powell and Mead (the latter pictured right), which together store Colorado River water for states including California, Arizona and Nevada.

Most projections suggest that the river's flow will fall by $6-20 \%$ by the middle of the century. A $10 \%$ drop would mean a $25 \%$ chance of reservoirs being fully depleted on an annual basis, and a $20 \%$ drop would result in a $50 \%$ risk. Drought risk rises steeply after 2026, but management interventions could do much to reduce the risk of reservoir depletion, say the researchers.

\section{INVERTEBRATE IMMUNITY}

\section{Infection in real time}

PLoS Pathog. 5, e1000518 (2009)

Scientists have a hard time following the initial action in an infection, but Will Wood at the University of Bath, UK, and his colleagues have tracked the early interactions between bacteria and immune cells as they battle for dominance in Drosophila embryos.
They found that non-pathogenic Escherichia coli are successfully ingested by haemocytes, phagocytic immune cells that travel throughout the developing fruitfly.

However, the pathogenic bacterium Photorhabdus asymbiotica causes the haemocytes to freeze in place. By inserting or deleting specific host and pathogen genes, the authors showed that the haemocytes froze only when they engulfed a bacterial toxin called Mcf1, which seemed to have an effect on the immune cell cytoskeleton.

\section{CHEMICAL BIOLOGY}

\section{0 million strong}

Nature Chem. Biol. doi:10.1038/nchembio.211 (2009) By tagging small molecules with short, doublestranded DNA fragments, Barry Morgan and his colleagues at GlaxoSmithKline in Waltham, Massachusetts, have created a collection of 800 million compounds that can be screened much faster than conventional chemical libraries.

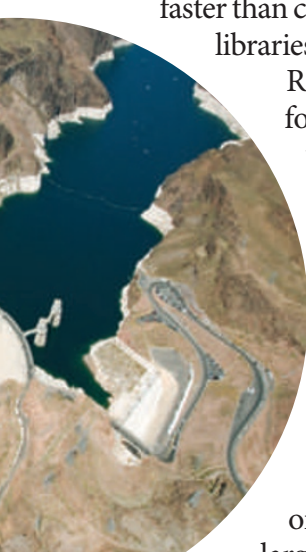

Researchers can screen for ligands that bind to a protein target and then identify the new molecule by sequencing the DNA 'barcode' attached. As a proof of concept, the authors probed their DNA-tagged library - which is at least two orders of magnitude larger than a typical small-molecule library — for enzyme-blocking drug leads and pulled out novel inhibitors of two kinases.

\section{STRUCTURAL BIOLOGY}

\section{Get into the groove}

Proc. Natl Acad. Sci. USA doi:10.1073/ pnas.0906532106 (2009).

A class of small molecules can distort the structure of DNA, disrupting its interaction with proteins that control gene expression.

Pyrrole-imidazole polyamides bind to the minor groove of the DNA double helix. David Chenoweth and Peter Dervan of the California Institute of Technology in Pasadena found that a cyclic polyamide binding in the minor groove compresses the major groove, which is where proteins called transcription factors bind DNA to regulate gene expression.

The polyamide also caused the helix to bend more than $18^{\circ}$ towards the major groove, another distortion that could interfere with transcription-factor binding.

\section{JOURNAL CLUB}

\section{Douglas Kell}

The University of Manchester, UK

\section{A systems biologist ponders how disparate ideas can sometimes come together beautifully.}

If $\mathrm{X}$ alone and $\mathrm{Y}$ alone cannot explain a phenomenon, sometimes together they can. As the late biochemist Henrik Kacser remarked: "To understand the whole you must look at the whole."

Prion diseases, for example, are closely associated with the conformational change of the prion protein PrP from its normal form to an aggregating, autocatalysing, pathologic form, $\mathrm{PrP}^{\mathrm{Sc}}$. But clumping prions don't tell the whole story. Their levels often correlate poorly with disease progression, and it is far from clear how a simple conformational change leads to the holes in brain tissue seen in late-stage disease.

It is also clear that poorly liganded iron is highly neurotoxic, mainly because it can spur the production of the highly reactive and toxic hydroxyl radical $\mathrm{OH}^{\circ}-$ heavily involved in the progression of many other degenerative diseases and ageing. Neena Singh at Case Western Reserve University in Cleveland, Ohio, and her colleagues have now tied these two disparate threads together.

$\mathrm{PrP}^{\mathrm{Sc}}$, they found, can sequester cellular iron in insoluble $\mathrm{PrP}^{\mathrm{Sc}}$ ferritin complexes, making it biounavailable, leading to increased iron uptake and an overall excess of iron in brain tissue (A. Singh et al., PLoS Pathog. 5, e1000336; 2009). Modified iron metabolism is found in both scrapie and sporadic Creutzfeldt-Jakob disease, and such work stresses that it is not only the total amount of Fe(II) and Fe(III) that matters but their speciation. It is yet to be shown whether PrP ${ }^{\mathrm{Sc}}$-ferritin complexes catalyse $\mathrm{OH}^{\circ}$ production directly, but if they do, this could account for the massive damage observed. Recognition of this could have a colossal effect on our thinking and provide new therapeutic (and dietary) options based on iron chelation for these and other syndromes.

Discuss this paper at http://blogs. nature.com/nature/journalclub 